Bull. Austral. Math. Soc.

Vol. 55 (1997) [311-319]

\title{
INSTABILITY OF THE DIRICHLET PROBLEM FOR HAMILTON-JACOBI EQUATION
}

\section{KEWEI ZHANG}

We show the instability of solutions of the Dirichlet problem for Hamilton-Jacobi equations under quite general conditions.

In this paper we show that under quite general conditions the solutions of the Dirichlet problem for the Hamilton-Jacobi equation

$$
\begin{cases}H(x, u(x), D u(x)) & =0, \text { in } \Omega \\ \left.u\right|_{\text {on }} & =u_{0}\end{cases}
$$

are unstable under $L^{\infty}$ external perturbations. We establish this result by constructing $W^{1, \infty}$ approximate solutions which converge in the weak-* sense to a given smooth subsolution. Let $\Omega \subset \mathbb{R}^{n}$ be an open, bounded and connected subset with Lipschitz boundary. We denote by $\partial \Omega$ and $\bar{\Omega}$ its boundary and closure respectively. Our main result is the following:

THEOREM 1. Suppose $H: \bar{\Omega} \times \mathbb{R} \times \mathbb{R}^{n} \mapsto \mathbb{R}$ is continuous and coercive; that is, for each compact subset $K$ of $\mathbb{R}, H(x, u, P) \rightarrow+\infty$ as $|P| \rightarrow \infty$ uniformly with respect to $(x, u) \in \bar{\Omega} \times K$. Suppose $u \in C^{1}(\Omega)$ is a subsolution of $(1)$ in the sense that $H(x, u(x), D u(x)) \leqslant 0$ in $\Omega$. Then there exists a bounded sequence $\left(u_{j}\right)$ in $W^{1, \infty}(\Omega)$, such that $\left.u_{j}\right|_{\theta n}=\left.u\right|_{\theta n}$,

$$
u_{j} \stackrel{*}{\rightarrow} u, \text { in } W^{1, \infty}(\Omega)
$$

and

$$
\left\|H\left(\cdot, u_{j}(\cdot), D u_{j}(\cdot)\right)\right\|_{L^{\infty}(\Omega)} \rightarrow 0
$$

as $j \rightarrow \infty$, where $\stackrel{*}{\rightarrow}$ denotes weak-* convergence.

Notice that $(3)$ is equivalent to the existence of a sequence $\left(h_{j}\right)$ in $L^{\infty}(\Omega)$, such that

$$
H\left(x, u_{j}(x), D u_{j}(x)\right)=h_{j}(x)
$$

almost everywhere in $\Omega$ and $h_{j} \rightarrow 0$ in $L^{\infty}(\Omega)$.

Received 23rd April, 1996

I would thank J. Kristensen and H. B. Thompson for many helpful suggestions.

Copyright Clearance Centre, Inc. Serial-fee code: 0004-9729/97 \$A2.00+0.00. 
REMARK 1. Theorem 1 implies that the solutions of the perturbed system

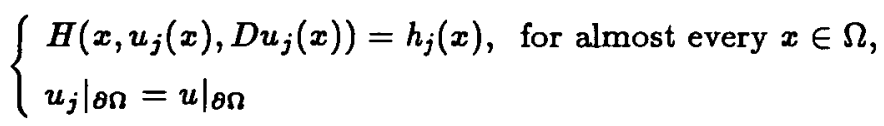

converge in $L^{\infty}$ norm to the given subsolution, rather than a solution of the original problem (1). Hence the system is not stable under $L^{\infty}$-perturbations.

REMARK 2. In Theorem 1, we do not assume convexity of $H$ in $P \in \mathbb{R}^{n}$. What we really need is a condition such that when $u \in \mathbb{R}$ is in a bounded set, $\{P, H(x, u, P) \leqslant 0\}$ is uniformly bounded. Existence results for (1) can be established under additional assumptions to those of Theorem 1 (see [9, Chapter 4], [2, Section 2.6]). Theorem 1 can be extended to the case $\Omega=\mathbb{R}^{n}$ if we assume further that the subsolution $u$ is uniformly continuous and bounded in $\mathbb{R}^{n}$ and $\boldsymbol{H}(\boldsymbol{x}, u, P) \rightarrow+\infty$ uniformly with respect to $(x, u) \in \mathbb{R}^{n} \times \mathbb{R}$.

This result improves on some earlier ones in $[6,7]$ where a variational approach was taken in some $L^{p}$ spaces under stronger hypotheses on $H$. There is a vast literature on the Hamilton-Jacobi equation and viscosity solutions of the equation (see $[9,2]$ and the references therein). These results are used mainly in the study of problems in control theory where $H(\cdot, u, P)$ is usually a Lipschitz function of $x$ so that the corresponding ordinary differential equation can be solved $[\mathbf{9}, \mathbf{2}])$. However, when the theories on existence, and uniqueness are applied to problems arising from, for example, the so called Shape-from-Shading in computer vision, the question of stability under $L^{\infty}$ external perturbations may occur and the viscosity solutions might not be the only preferred solutions for the problem. The shape from shading comes as an inverse problem. The question is whether we can recover the shape of a graph from its shading under one single light source (see [8] and the references therein), or what the possible shapes of the object are provided that the shading information is given. For inverse problems, an important issue is the stability of solutions under reasonably possible perturbations of the system. In the shape-from-shading problem, the possibility of small $L^{\infty}$ perturbations cannot be ruled out because of errors in the measurements and other noise factors. $[11,10]$ proposed the use of viscosity solutions of HamiltonJacobi equations as the possible solutions because under some delicate conditions on the shading function (for example, in the case of the eikonal equation (4) below, the function $\mathcal{E}$ has only one zero point in the domain, see [10, Theorem 1]) the viscosity solution is unique or the solutions can be classified. However, viewing the approximate solutions given by Theorem 1 for the eikonal equation (4) indicates that the shading of the approximate graph is almost the same as that of an exact $C^{1}$-solution [4]. [5] provides a possible application of shape from shading to the recovery of the shape of a particular area on the Mars from two pictures taken by a spaceship far away (about 
$1500 \mathrm{~km}$ ) from Mars and the area is comparatively small (about $33.1 \times 26.5 \mathrm{~km}$ ). The issue of stability turns out to be very important for this case. I doubt that viscosity solutions based on the photograph could give the right shape in this case. Therefore, it is natural to consider all the possible solutions under small perturbations, rather than viscosity solutions alone. For the case of eikonal equation (4) applied to the shape from shading problem, Theorem 1 implies that all the smooth subsolutions are possible shapes for the given image up to some noise.

In $[6,4]$, the instability for the eikonal equation

$$
|D u(x)|=\mathcal{E}(x)
$$

was established. In [4], we further exhibited, by using some viewing software, the graphs of the exact solutions of (4) and those of the approximate solutions based on subsolutions. Under the given viewing conditions assumed by the model, we cannot tell the difference between the shading given by an exact solution and an approximate solution while the latter is much closer to a subsolution in $C(\Omega)$ than to the exact solution. This is the main motivation of the present work for the Hamilton-Jacobi equation in its general form (1).

We need some preparatory notation and results for the proof of Theorem 1. Let $e_{1}, \ldots, e_{n}$ be the canonical basis for $\mathbb{R}^{n}$. Let $L^{p}(\Omega)(1 \leqslant p \leqslant \infty)$ be the usual Lebesgue spaces. We denote by $C^{k}(\Omega)(k=0,1,2 \ldots)$ the restrictions of $k$-continuously differentiable functions defined in $\mathbb{R}^{n}$ to $\bar{\Omega}$. We let $C^{0}(\Omega)=C(\Omega)$ and $W^{1, p}(\Omega), 1 \leqslant p \leqslant \infty$, denote the usual Sobolev space. Since $\partial \Omega$ is Lipschitz, elements of $W^{1, p}(\Omega)$ admit traces on $\partial \Omega$ (see [1]). We also define $W_{0}^{1, p}(\Omega)$, as usual, by

$$
W_{0}^{1, p}(\Omega)=\left\{u \in W^{1, p}(\Omega), \text { the trace on } \partial \Omega \text { of } u \text { is } 0\right\} .
$$

For a function $u: \Omega \rightarrow \mathbb{R}$, let $D u(x)$ be the gradient of $u$ at $x \in \Omega$. If $v: \Omega \rightarrow \mathbb{R}^{n}$ is a vector valued function such that each of its components is in $C^{k}(\Omega)\left(L^{p}(\Omega)\right.$, respectively), we say that $v$ belongs to $C^{k}\left(\Omega ; \mathbb{R}^{n}\right)\left(L^{p}\left(\Omega, \mathbb{R}^{n}\right)\right.$, respectively). Weak convergence in $W^{1, p}(\Omega)$ is denoted by $\rightarrow$ and strong convergence by $\rightarrow$.

Our construction of the approximate solutions is based on some simple observations on Young measures (see, for example, $[3,12]$ ).

LemMA 1. Let $\left\{z_{j}\right\}$ be a bounded sequence in $L^{\infty}\left(\Omega ; \mathbb{R}^{s}\right)$. Then there exist a subsequence $\left\{z_{j_{k}}\right\}$ of $\left\{z_{j}\right\}$ and a family $\left\{\nu_{x}\right\}, x \in \Omega$, of probability measures on $\mathbb{R}^{*}$ (Young measures), depending measurably on $x \in \Omega$, such that

$$
f\left(z_{j_{k}}\right) \stackrel{*}{\rightarrow}\left\langle\nu_{x}, f(\cdot)\right\rangle=\int_{\mathbb{R}^{*}} f(\lambda) d \nu_{x}(\lambda) \quad \text { in } L^{\infty}(\Omega)
$$


for every continuous function $f: \mathbb{R}^{\boldsymbol{e}} \rightarrow \mathbb{R}$.

In order to find some necessary conditions for possible approximate sequences, we associate with (1) a functional given by

$$
I(u)=\int_{\Omega}|B(x, u(x), D u(x))| d x .
$$

The following is a simplified version of a result established in [7, Proposition 1].

Lemma 2. Suppose $H$ satisfies the assumptions of Theorem 1. We further assume that $H(x, u, P)$ is convex in $P \in \mathbb{R}^{n}$ for each fixed $(x, u) \in \bar{\Omega} \times \mathbb{R}$. Suppose that there exists a sequence $\left(u_{j}\right)$ in $W^{1, \infty}(\Omega)$ such that

$$
\lim _{j \rightarrow \infty} \int_{\Omega}\left|H\left(x, u_{j}(x), D u_{j}(x)\right)\right| d x=0 .
$$

Then up to a subsequence

$$
u_{j} \rightarrow u \text { in } W^{1, \infty}(\Omega)
$$

and

$$
H(x, u(x), D u(x)) \leqslant 0 \text { almost everywhere in } \Omega .
$$

If $\left\{\nu_{x}\right\}_{x \in \Omega}$, is the family of Young measures corresponding to $\left\{D u_{j}\right\}$, then

$$
\operatorname{supp} \nu_{x} \subset\{P ; H(x, u(x), P)=0\}
$$

for almost every $x \in \Omega$, where supp $\nu_{x}$ is the support of $\nu_{x}$ in $\mathbb{R}^{n}$.

To conclude our preparation, we state a simple result in real analysis.

Lemma 3. Suppose $\Omega \subset \mathbb{R}^{n}$ is a bounded and open subset. Then there exists a sequence of open hypercubes $\left(D_{k}\right), k=1,2, \ldots$ with side length $2^{-j_{k}}$ for some positive integer $j_{k}$ and with their edges parallel to the coordinate axes, such that $D_{k} \cap D_{s}=\emptyset$ and

$$
\Omega \subset \bigcup_{k=1}^{\infty} \overline{D_{k}} \subset \bar{\Omega} .
$$

PROOF OF THEOREM 1: Our construction of the approximate sequence is based on the observation on the Young measures (7). If we write $u_{j}$ in Lemma 2 as $u+\phi_{j}$, where $u$ is the smooth subsolution of $(1)$, we see that $\phi_{j} \stackrel{*}{\rightarrow} 0$. Let $\left\{\bar{\nu}_{x}\right\}_{x \in \Omega}$ be the family of Young measures corresponding to $\left(D \phi_{j}\right)$. We have that

$$
\operatorname{supp} \bar{\nu}_{x} \subset H_{x, u(x)}:=\left\{\lambda \in \mathbb{R}^{n}, H(x, u(x), D u(x)+\lambda)=0\right\}
$$


almost everywhere in $\Omega$.

Notice that the origin 0 of $\mathbb{R}^{n}$ is always in the set $H_{x, u(x)}$ or in

$$
G_{x, u(x)}=\left\{\lambda \in \mathbb{R}^{n}, H(x, u(x), D u(x)+\lambda)<0\right\} .
$$

In order to construct a simple sequence such that the gradients of the functions generate such a Young measure, we pick up $2^{n}$ vectors on $\partial H_{x, u(x)}$.

Let $\mathcal{D}_{1}=\left\{D_{k}^{(1)}\right\}_{k=1}^{J(1)}$ be a decomposition of $\Omega$ by hypercubes given by Lemma 3 . $J(1)$ can be a finite positive integer or $+\infty$. We define $\mathcal{D}_{2}=\left\{D_{k}^{(2)}\right\}_{k=1}^{J(2)}$ by dividing each $D_{k}^{(1)}$ evenly into $2^{n}$ smaller hypercubes. Therefore $\mathcal{D}_{2}$ is also a decomposition of $\Omega$. Suppose that for some integer $j>1$, we can define $\mathcal{D}_{j}=\left\{D_{k}^{(j)}\right\}_{k=1}^{J(j)}$, we then define $\mathcal{D}_{j+1}$ by dividing each $D_{k}^{(j)}$ evenly into $2^{n}$ hypercubes. Thus we can define a sequence of decompositions $\mathcal{D}_{j}$ by induction. Let

$$
D_{k}^{(j)}=\left\{x=\left(x_{1}, x_{2}, \ldots, x_{n}\right), x_{k, s}^{(j)}<x_{s}<x_{k, \mathrm{~s}}^{(j)}+h_{k}^{(j)}, s=1,2, \ldots, n\right\},
$$

where $h_{k}^{(j)}>0$. We denote by $c_{k}^{(j)}$ the central point of $D_{k}^{(j)}$. If we set

$$
h_{j}=\sup _{1 \leqslant k \leqslant J(j)}\left\{h_{k}^{(j)}\right\},
$$

from our construction of $\mathcal{D}_{j}$ and the fact that $\Omega$ is bounded, we see that $h_{j} \rightarrow 0$ as $j \rightarrow \infty$. We consider two different cases.

CASE 1. $H\left(c_{k}^{(j)}, u\left(c_{k}^{(j)}\right), D u\left(c_{k}^{(j)}\right)\right)=0$. In this case we define

$$
\phi_{k}^{(j)}(x) \equiv 0 \quad \text { for } x \in \overline{D_{k}^{(j)}} .
$$

CASE 2. $H\left(c_{k}^{(j)}, u\left(c_{k}^{(j)}\right), D u\left(c_{k}^{(j)}\right)\right)<0$. In this case, we let

$$
A_{k, s,+}^{(j)}=a_{k, s,+}^{(j)} e_{s}, A_{k, s,-}^{(j)}=a_{k, \Omega,-}^{(j)} e_{s}, s=1, \ldots, n
$$

be vectors in $\mathbb{R}^{n}$ such that

$$
\begin{aligned}
& a_{k, a,+}^{(j)}=\inf _{i>0}\left\{t, H\left(c_{k}^{(j)}, u\left(c_{k}^{(j)}\right), D u\left(c_{k}^{(j)}\right)+t e_{s}\right) \geqslant 0\right\}, \\
& a_{k, \Omega,-}^{(j)}=\sup _{t<0}\left\{t, H\left(c_{k}^{(j)}, u\left(c_{k}^{(j)}\right), D u\left(c_{k}^{(j)}\right)+t e_{s}\right) \geqslant 0\right\} .
\end{aligned}
$$

From our coercivity condition on $H$, boundedness of $u$ in $\bar{\Omega}$ and a simple compactness argument, we see that $a_{k, \Omega,+}^{(j)}>0$ and $a_{k, \ell,-}^{(j)}<0$ both exist and are uniformly bounded, say, by some finite $M>0$ for all $j=1,2, \ldots$. 
Now we define, in $\overline{D_{k}^{(j)}}$, the following affine functions

$$
\begin{aligned}
& f_{k, s,+}^{(j)}(x)=a_{k, s,+}^{(j)}\left(x_{s}-x_{k, s}^{(j)}\right), \\
& f_{k, s,-}^{(j)}(x)=a_{k, s,-}^{(j)}\left[x_{s}-\left(x_{k, s}^{(j)}+h_{k}^{(j)}\right)\right],
\end{aligned}
$$

for $s=1,2, \ldots, n$. Notice that both $f_{k, s,+}^{(j)}$ and $f_{k, s,-}^{(j)}$ are nonnegative affine functions in $\overline{D_{k}^{(j)}}$ and they vanish only on

$$
\left\{x \in \overline{D_{k}^{(j)}}, x_{s}=x_{k, s}^{(j)}\right\} \text { and }\left\{x \in \overline{D_{k}^{(j)}}, x_{s}=x_{k, s}^{(j)}+h_{k}^{(j)}\right\}
$$

respectively. We then define

$$
\phi_{k}^{(j)}(x)=\min _{1 \leqslant s \leqslant n}\left\{\min \left\{f_{k, s,+}^{(j)}(x), f_{k, s,-}^{(j)}(x)\right\}\right\}
$$

in $\overline{D_{k}^{(j)}}$. It is easy to see that $\phi_{k}^{(j)}$ is a piecewise affine function in $\overline{D_{k}^{(j)}}$ and $\left.\phi_{k}^{(j)}\right|_{\partial D_{k}^{(j)}}$ $=0$. We extend $\phi_{k}^{(j)}$ to be defined in $\bar{\Omega}$ by zero outside $D_{k}^{(j)}$.

Combining both cases, we define

$$
\phi_{j}(x)=\sum_{k=1}^{J(j)} \phi_{k}^{(j)}(x)
$$

in $\Omega$. It is easy to see that $\phi_{j}$ belongs to $W_{0}^{1, \infty}(\Omega)$. We seek to prove that the sequence $\left(\phi_{j}\right)$ satisfies

$$
\begin{aligned}
& \text { (a) } \phi_{j} \stackrel{*}{*} 0 \text { as } j \rightarrow \infty \\
& \text { (b) }\left\|H\left(\cdot, u(\cdot)+\phi_{j}(\cdot), D u(\cdot)+D \phi_{j}(\cdot)\right)\right\|_{L^{\infty}(\Omega)} \rightarrow 0 \text { as } j \rightarrow \infty
\end{aligned}
$$

To prove (a), we first consider $\left\|\phi_{j}\right\|_{L^{\infty}(\Omega)}$. We have, from the fact that $\phi_{k}^{(j)}(x) \phi_{l}^{(j)}(x)$ $=0$ whenever $k \neq l, 1 \leqslant k, l \leqslant J(j)$, that

$$
\begin{aligned}
\left\|\phi_{j}\right\|_{L^{\infty}(\Omega)} & =\left\|\sum_{k=1}^{J(j)} \phi_{k}^{(j)}\right\|_{L^{\infty}(\Omega)} \leqslant \sup _{1 \leqslant k \leqslant J(j)}\left\|\phi_{k}^{(j)}\right\|_{L^{\infty}\left(D_{k}^{(j)}\right)} \\
& \leqslant \sup _{1 \leqslant k \leqslant J(j)} \max _{1 \leqslant s \leqslant n}\left\{\max \left\{f_{k, s,+}^{(j)}(x), f_{k, s,-}^{(j)}(x)\right\}\right\} \\
& \leqslant \sup _{1 \leqslant k \leqslant J(j)} M h_{k}^{(j)} \leqslant M h_{j} .
\end{aligned}
$$

Since $h_{j} \rightarrow 0$, we see that $\left\|\phi_{j}\right\|_{L^{\infty}(\Omega)} \rightarrow 0$ as $j \rightarrow \infty$. 
Since

$$
\begin{aligned}
\left\|D \phi_{j}\right\|_{L^{\infty}(\Omega)} & \leqslant \sup _{1 \leqslant k \leqslant J(j)}\left\|D \phi_{k}^{(j)}\right\|_{L^{\infty}\left(D_{k}^{(j)}\right)} \\
& \leqslant \sup _{1 \leqslant k \leqslant J(j)} \max _{1 \leqslant s \leqslant n}\left\{\max \left\{\left|a_{k, s,+}^{(j)}\right|,\left|a_{k, s,-}^{(j)}\right|\right\}\right\} \leqslant M,
\end{aligned}
$$

we see that $\left(\phi_{j}\right)$ is a bounded sequence in $W_{0}^{1, \infty}(\Omega)$. If $\left(\phi_{j}\right)$ does not converge in the weak-* sense to 0 in $W_{0}^{1, \infty}(\Omega)$, there exists a subsequence $\left(\phi_{j_{m}}\right)$ converging in the weak-* sense to some $\phi_{0}$ in $W_{0}^{1, \infty}(\Omega)$ and $\phi_{0}$ is not identically zero in $\Omega$. This contradicts the fact that $\phi_{j} \rightarrow 0$ uniformly in $\bar{\Omega}$. (a) is proved. To prove (b), we have

$$
\begin{aligned}
& \left\|H\left(\cdot, u(\cdot)+\phi_{j}(\cdot), D u(\cdot)+D \phi_{j}(\cdot)\right)\right\|_{L^{\infty}(\Omega)} \\
& \leqslant \sup _{1 \leqslant k \leqslant J(j)}\left\{\left\|H\left(\cdot, u(\cdot)+\phi_{j}(\cdot), D u(\cdot)+D \phi_{j}(\cdot)\right)\right\|_{L^{\infty}\left(D_{k}^{(j)}\right)}\right\} \\
& \leqslant \sup _{1 \leqslant k \leqslant J(j)}\left\{\left\|H\left(\cdot, u(\cdot)+\phi_{j}(\cdot), D u(\cdot)+D \phi_{j}(\cdot)\right)-H\left(\cdot, u(\cdot), D u(\cdot)+D \phi_{j}(\cdot)\right)\right\|_{L^{\infty}\left(D_{k}^{(j)}\right)}\right\} \\
& +\sup _{1 \leqslant k \leqslant J(j)}\left\{\left\|H\left(\cdot, u(\cdot), D u(\cdot)+D \phi_{j}(\cdot)\right)\right\|_{\left.L^{\infty}\left(D_{k}^{(j)}\right)\right\}}\right\} \\
& =I_{j}^{(1)}+I_{j}^{(2)}
\end{aligned}
$$

where

$$
\begin{aligned}
I_{j}^{(1)}=\sup _{1 \leqslant k \leqslant J(j)}\left\{\| H\left(\cdot, u(\cdot)+\phi_{j}(\cdot), D u(\cdot)\right.\right. & \left.+D \phi_{j}(\cdot)\right) \\
& -H\left(\cdot, u(\cdot), D u(\cdot)+D \phi_{j}(\cdot)\right) \|_{\left.L^{\infty}\left(D_{k}^{(j)}\right)\right\} .}
\end{aligned}
$$

Since $H$ is continuous in $\bar{\Omega} \times \mathbb{R} \times \mathbb{R}^{n}, u$ belongs to $C^{1}(\bar{\Omega}),\left\|D \phi_{j}\right\|_{L^{\infty}\left(D_{k}^{(j)}\right)}$ is bounded by $M$ and $\phi_{j} \rightarrow 0$ uniformly in $\bar{\Omega}$, we see that

$$
\lim _{j \rightarrow \infty} I_{j}^{(1)}=0 .
$$

We now have

$$
\begin{aligned}
I_{j}^{(2)}= & \sup _{1 \leqslant k \leqslant J(j)}\left\{\left\|H\left(\cdot, u(\cdot), D u(\cdot)+D \phi_{k}^{(j)}(\cdot)\right)\right\|_{L^{\infty}\left(D_{k}^{(j)}\right)}\right\} \\
\leqslant & \sup _{1 \leqslant k \leqslant J(j)}\left\{\| H\left(\cdot, u(\cdot), D u(\cdot)+D \phi_{k}^{(j)}(\cdot)\right)\right. \\
& \left.\quad-H\left(c_{k}^{(j)}, u\left(c_{k}^{(j)}\right), D u\left(c_{k}^{(j)}\right)+D \phi_{k}^{(j)}(\cdot)\right) \|_{L^{\infty}\left(D_{k}^{(j)}\right)}\right\} \\
& +\sup _{1 \leqslant k \leqslant J(j)}\left\{\left\|H\left(c_{k}^{(j)}, u\left(c_{k}^{(j)}\right), D u\left(c_{k}^{(j)}\right)+D \phi_{k}^{(j)}(\cdot)\right)\right\|_{L^{\infty}\left(D_{k}^{(j)}\right)}\right\} \\
= & K_{1}^{(j)}+K_{2}^{(j)},
\end{aligned}
$$


where

$$
K_{2}^{(j)}=\sup _{1 \leqslant k \leqslant J(j)}\left\{\left\|H\left(c_{k}^{(j)}, u\left(c_{k}^{(j)}\right), D u\left(c_{k}^{(j)}\right)+D \phi_{k}^{(j)}(\cdot)\right)\right\|_{L^{\infty}\left(D_{k}^{(j)}\right)}\right\}=0
$$

because

$$
D \phi_{k}^{(j)}(x) \in\left\{a_{k, s,+}^{(j)}, a_{k, e,-}^{(j)}, 1 \leqslant s \leqslant n\right\}
$$

almost everywhere in $D_{k}^{(j)}$ and

$$
H\left(c_{k}^{(j)}, u\left(c_{k}^{(j)}\right), D u\left(c_{k}^{(j)}\right)+a_{k, 8, \pm}^{(j)} e_{s}\right)=0
$$

for all $k, s, j$; while

$$
\begin{aligned}
K_{1}^{(j)}= & \sup _{1 \leqslant k \leqslant J(j)}\left\{\| H\left(\cdot, u(\cdot), D u(\cdot)+D \phi_{k}^{(j)}(\cdot)\right)\right. \\
& -H\left(c_{k}^{(j)}, u\left(c_{k}^{(j)}\right), D u\left(c_{k}^{(j)}\right)+D \phi_{k}^{(j)}(\cdot)\right) \|_{\left.L^{\infty}\left(D_{k}^{(j)}\right)\right\}} \\
\leqslant & \sup _{1 \leqslant k \leqslant J(j)} \max _{1 \leqslant s \leqslant n}\left\{\| H\left(\cdot, u(\cdot), D u(\cdot)+a_{k, s,+}^{(j)} e_{s}\right)\right. \\
& -H\left(c_{k}^{(j)}, u\left(c_{k}^{(j)}\right), D u\left(c_{k}^{(j)}\right)+a_{k, s,+}^{(j)}\right) \|_{L^{\infty}\left(D_{k}^{(j)}\right)}, \\
& \| H\left(\cdot, u(\cdot), D u(\cdot)+a_{k, s,-}^{(j)} e_{s}\right) \\
& \left.-H\left(c_{k}^{(j)}, u\left(c_{k}^{(j)}\right), D u\left(c_{k}^{(j)}\right)+a_{k, s,-}^{(j)} e_{s}\right) \|_{L^{\infty}\left(D_{k}^{(j)}\right)}\right\}
\end{aligned}
$$

hence $K_{1}^{(j)} \rightarrow 0$ as $j \rightarrow \infty$, because $\left\{\left|a_{k, \varepsilon, \pm}^{(j)}\right|\right\}$ is bounded by $M, H$ is uniformly continuous in every bounded set of $\bar{\Omega} \times \mathbb{R} \times \mathbb{R}^{n}$ and the side length $h_{k}^{(j)}$ of $D_{k}^{(j)}$ is bounded by $h_{j}$ which converges to 0 as $j \rightarrow \infty$.

\section{References}

[1] R.A. Adams, Sobolev spaces (Academic Press, New York, 1975).

[2] G. Barles, Solutions de viscosité des équations de Hamilton-Jacobi (Springer-Verlag, Berlin, Heidelberg, New York, 1994).

[3] J.M. Ball, 'A version of the fundamental theorem for Young measures', in Lecture Notes in Physics 344 (Springer-Verlag, Berlin, Heidelberg, New York, 1988), pp. 207-215.

[4] I. Barnes, K.-W. Zhang, 'Instability of the eikonal equation and shape from shading', (in preparation).

[5] M.J. Carlotto, 'Digital imagery analysis of unusual Martian surface features', Appl. Optics 27 (1988), 1926-1933. 
[6] J. Chabrowski, K.-W. Zhang, 'On shape from shading problem', in Functional Analysis, Approximation Theory and Numerical Analysis, (J.M. Rassias, Editor) (World Scientific, 1994), pp. 93-105.

[7] J. Chabrowski, K.-W. Zhang, 'On variational approach to the Hamilton-Jacobi PDE', Comment. Math. Univ. Carolin. 34 (1993), 613-633.

[8] B.K.P. Horn and M.J. Brooks, Shape from shading, (B.K.P. Horn and M.J. Brooks, Editors), Artificial Intelligence (MIT Press, 1989).

[9] P.L. Lions, Generalized solutions of Hamilton-Jacobi equations (Pitman, London, 1982).

[10] P.L. Lions, E. Rouy, A. Tourin, 'Shape-from-shading, viscosity solutions and edges', $\mathrm{Nu}$ mer. Math. 64 (1993), 323-353.

[11] E. Rouy, A. Tourin, 'A viscosity solution approach to shape-from-shading', SIAM $J$. Numer. Anal. 29 (1992), 867-884.

[12] L. Tartar, 'Compensated compactness and applications to partial differential equations', in Nonlinear Analysis and Mechanics, (R. Knops, Editor), Heriot-Watt Symposium IV (Pitman, 1979), pp. 136-212.

Department of Mathematics

Heriot-Watt University

Richarton, Edinburgh EH14 4AS

United Kingdom

e-mail: kewei@ma.hw.ac.uk 